\title{
19. The Detection of Soft X-Rays with Charged Coupled Detectors
}

\author{
P. Burstein and J. M. Davis \\ American Science and Engineering, Inc., Fort Washington \\ Cambridge, MA 02139, USA
}

The characteristics of an ideal soft $x$-ray imaging detector are enumerated. of recent technical developments the CCD or charge coupled device goes furthest to meeting these requirements. Several properties of CCDs are described with reference to experimental work and their application to practical instruments is reviewed.

\subsection{Introduction}

The development of soft $x$-ray sensitive, electronic imaging detectors for scientific applications is a major concern of laboratories worldwide. In contrast to purely imaging applications scientific observations require quantitative, intensity and position information from the image. Therefore the characteristics of an ideal detector must include:

(1) A spatial resolution comparable to photographic film which implies a format with a large number of picture elements. In this case a large number is of the order $10^{6}$ to $10^{7}$.

(2) A high sensitivity, to achieve the efficient detection of single, incident photons, to minimize the degradation caused by system noise and to provide high temporal resolution.

(3) A stable transfer function between input and output in order to achieve a photometric accuracy of $1 \%$.

(4) Broad spectral response, or quantum efficiency, covering the energy range from 0.2 to $20 \mathrm{keV}$ coupled with energy resolution for single photons over the same range.

The search for a single detector which completely satisfies all these conditions has been largely unsuccessful. It has included imaging proportional counters [19.1], which combine very large areas, good energy resolution, but only moderate spatial resolution, microchannel plates with a variety of readout systems which have large areas, good spatial resolution but extremely limited energy resolution $[19.2,3]$, and more recently and quite promisingly charge coupled devices or CCDs.

In the following paragraphs the properties of CCDs as they apply to soft $x$-ray detection and their application to scientific investigations is discussed.

\subsection{Properties of Charge Coupled Devices}

CCDs are closely spaced, two-dimensional arrays of MOS capacitors which are laid down on a silicon substrate, shown schematically in Fig.19.1. The capacitors are electrically isolated from each other by the $p$ - and n-type archi- 
(FRONT-SIOE ILLUMINATEO)

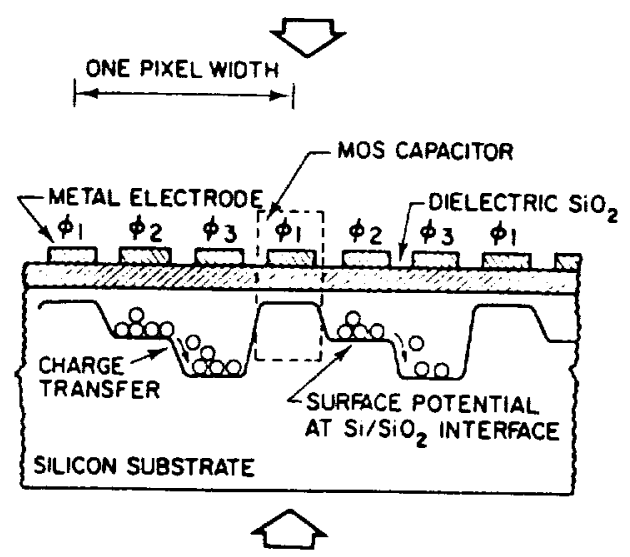

Fig.19.1 Functional description of a typical three-phase CCD architecture illustrating front and back il lumination, taken from MELEN and BUSS [19.7]

(BACK-SIDE ILLUMINATEO)

tecture of the device and by the applied voltages. A photosite, or picture element (pixel), consists of a set of three adjacent capacitors grouped in the columns of the array. The electrodes of the capacitors are independently controlled by "clock" or "gate" voltages. Because of this arrangement this type of CCD is known as a three-phase device.

When an incident $x$ ray is absorbed in the silicon substrate it excites electrons into the conduction band which then diffuse into a depletion layer formed by the positive voltages applied to the electrodes. These applied voltages form a potential well which traps charge at a particular photosite. To read out the device the applied valtages are changed, or clocked, so that the charge at each photosite along a row is transferred vertically to the adjacent site in its column. The charges in the row formed by the lowest site in each column are transferred into a shift register where they are read out serially following on-chip amplification.

The key to the operation of the CCO as an $x$-ray detector is the use of the interaction site as the storage site. It can be thought of as an array of solid-state detectors each with its own memory for the CCO can accept photons over its entire surface simultaneously.

In "conventional $x$-ray detection" by the CCO [19.4,5] the detector is used exactly as for visible light detection, as a total energy detector. The output of a particular pixel is a charge which is proportional to the total amount of energy deposited in the pixel.

The spatial resolution can be determined largely on the basis of pixelto-pixel spacing and charge localization between pixels.

When only one $x$-ray photon is known to have interacted in an element of the array, then the charge in that element will be a function of the photon energy, as in a solid-state detector. Thus, each pixel will have an associated energy to charge transfer curve, and hence an energy resolution curve. The energy resolution, to a first approximation, is that of a tiny solid 
On the average one electron-hole pair is created in the pixel for every $3.6 \mathrm{eV}$ of energy in the $x$-ray photon. This is a very small amount of energy when compared with other soft $x$-ray detectors. Thus, a proportionally greater number of electrons will be created for each photon interaction, and the associated Poisson (or Poisson-like) statistics become more precise.

Since all pixels have a similar type of response, the CCD may be used as a nondispersive $x$-ray spectrometer $[19.5,6]$. The on ly requirement is that the probability of any single pixel receiving more than one photon during an integration period be small.

CCOs have several important characteristics which influence their operation as $x$-ray imaging detectors which we will briefly describe. First the quantum efficiency, defined as the probability of detecting an incident photon is a function of $x-r a y$ energy and is close to unity for energies between 1 and 10 kilovolts. At the higher energies the efficiency falls off ween 1 ans as through the device without interacting while at the because the photons pass through the device without interacting illuminated in in the so-called front-iting structures on the front surface. These structures form a dead layer between 0.5 to 2 microns thick, and to overcome their effect CCDs have been operated in a back-illuminated mode. In this case the silicon substrate is illuminated directly, and to maximize the effase the silicon subs is tailored to the particular application, a process known as thinning. This leads to a consideratile improvement in sensitivity, and we have been able to detect carbon $K_{a} x$ rays at $250 \mathrm{eV}$ with RCA CCOS manufactured in this fashion.

An alternative approach has been developed by Texas Instruments, who have developed a virtual phase CCO $[19.8,9]$. In this device the three applied developed a vire tential is created through the use of ion implants in the $n$-type buried channel. By reducing the number of polysilicon gates per pixel from three to one, the thickness of the dead layer can be substantially reduced. However, ine, the thickness unable to detect in our tests of such a front-illuminated the modification is not an adequate carbon $k_{i 2} \times$ rays, and so in this respe

The intrinsic noise of a CCD limits both the energy resolution and the length of time a picture can be integrated. Noise levels of 30 electrons rms can be achieved corresponding to energy resolution of $250 \mathrm{eV}$. The energy $c$ an be achieved corresponding to energy energy [19.10], and therefore CCDS resolution is essentially independent of energy 19.10$]$, and therefore cCDs are better than proportional counters at energies above $500 \mathrm{eV}$ and marginal-
ly worse at lower energies. The ultimate noise goal is of the order of 10 electrons rins, which would be set by the stray capacitance of a few hundred pFs between the on-chip preamplifier and the last transfer gate. Noise levels approaching this have been reported in the literature [19.11] which makes their energy resolution superior to proportional counters.

To achieve these noise levels, the $C C D$ and the on-chip amplifier have been cooled. Typical operating temperatures are around $-100^{\circ} \mathrm{C}$. However, the operating temperature can be made too low for there are other completing operating temperature can important are the charge transfer efficiency ano the leakage current, which are also functions of temperature. The charge transfer efficiency is the fraction of the original charge transferred from transfer efficing incomple charge transfer results in a loss of both photometric accuracy and dynamic range and introduces smearing of the image. The leakage current is a measure of the charge of 
the spills from an illuminated pixel to adjacent dark pixels. In our tests with RCA CCDs we have found that an ideal operating temperature must be determined for each device, which is warm enough so that charge transfer ef ficiency is adequate ( 20.99995 to 0.99999$)$ while still cool enough to keep the leakage current acceptably low. This tradeoff has to be made on a device by device basis.

In principle the dynamic range of the CCO is limited by the readout noise and the full well capacity. The latter scales roughly as the pixel area, and for $30 \mu \mathrm{m}$ square pixels the well capacity is 2250,000 electrons. The typical dc level for the three-phase CCDs we have tested is several hundred electrons, which corresponds to a dynamic range of the order of or less than $10^{3}$.

Although one should be able to extend the dynamic range by improving the noise characteristics of the preamplifier this is not necessarily true in the $x$-ray region. For a single $x$-ray photon produces a large number of electrons,e.g.,a one kilovolt $x$ ray will contribute in excess of 300 electrons, and if this value is greater than the noise it will place the limit on the dynamic range which is thus energy dependent varying inversely with the incident photon energy.

Finally, CCDs have excellent linearity to increases in the incident $x$-ray intensity characteristics of solid-state detectors and pixel nonuniformities arising from processing variations and mask alignment errors during fabrication are generally quite small.

\subsection{Applications}

Although the primary incentive for our studies of CCDs has been their application to astronomical observations there are many other scientific investigations in which their sensitivity and excellent spatial resolution can be used to advantage. Examples are the in vivo examination, in real time, of biological specimens and the recording of the $x$-ray emission arising during the collapse of the fuel pellets used in inertial confinement fusion experiments.

In $x$-ray astronomy a heavy emphasis has been placed on obtaining observations with the highest spatial resolution. In this regard most electronic detectors have compared poorly to film. However, since film requires recovery which is impossible in most missions, astronomers have had to be satisfied with observations which were limited by the detector. As a numerical example, if we require one arc second resolution and we use a CCD with 15 um pixels, a focal length in excess of $6 \mathrm{~m}$ is required for the optical system. Such arguments have lead to the choice of a $10 \mathrm{~m}$ focal length for the Advanced $x$-ray Astronomical facility. The increased image size resulting from a large focal length is something of a mixed blessing. First the field of view subtended by the detector is correspondingly reduced. For instance an $800 \times 800$ array of 1 arc second pixels subtends a field of just over 13 arc minutes. This can be compared with the diameter of the sun which is 32 arc minutes. Secondly, such an image contains a tremendous amount of information which has to be processed digitally. If the intensity scale is divided into 256 gray levels, the number of bits required to specify the image is in excess of half a million. Thus unless very high data rates are available, the transmission time for the image can be very much greater than the exposure time. This difficulty tends to negate the advantage provided by the high sensitivity of the $\mathrm{CCO}$ which is roughly three orders of magnitude better 
than photographic emulsions and, for solar observation, allows exposure than photograph to a few tens of milliseconds. In principle this should open up a new field of coronal observations involving the dynamics of the coronal structures, changes at boundaries due to magnetic reconnection and the mechanisms of flaring events at high spatial resolutions. However, because of the high telemetry rates that are required the full potential of these studies has yet to be realized.

An added advantage of the CCO which results directly from its ability to detect single $x$-ray photons is its ability to minimize the effects of blooming. Blooming is the spreading of charge which has accumulated in overexposed areas to adjacent pixels of the CCD and is comon to all electronic imaging systems. In many astronomical applications, the source object has a very large dynamic range and thus the average flux from the region might dictate an optimum exposure which causes blooming somewhere else on the chip. Howaner, if the CCD is used in the single photon or spectrometric mode, many short exposures which will not cause blooming can be sumed electronically, without loosing spatial resolution because of the digital nature of the device, to provide a single image with an effective dynamic range larger than that of the CCD.

\subsection{Conclusions}

CCDs used for the detection of soft $x$ rays are a relatively new technology. They hold great promise as astronomical $x$-ray imaging detectors combining high spatial resolution and energy sensitivity. In practical applications cCOs have both advantages and disadvantages over competing technologies. On the plus side, they are compact, low-power devices whose operation requires neither the use of high voltages or hard vacuums. On the negative side they have to be cooled to temperatures on the order of $-100^{\circ} \mathrm{C}$ for optimum performance.

It is almost certain that in the next few years they will see wide application in a variety of space missions and the ir success will determine their future development.

\section{Acknow ledgements}

This work has been supported by NASA through several contracts.

\section{References}

19.1 P. Gorenstein, H. Gursky, F.R. Harnden, Jr., A. DeCaprio, and P. Bjorkoh Im: IEEE Trans. NS-22, 616 (1975)

19.2 R. Giacconi et al.: Astrophys. J. 230, 540 (1979)

19.3 J.M. Davis, J.k. Ting, and M. Gerassimenko: Space Sci. Instrum. 5 , 17 (1979)

19.4 G. Renda and J.L. Lowrance: Proc. Symp. on CCD Technology, JPL SP 43-21, 91 (1975)

19.5 P. Burstein, A.S. Krieger, M.J. Vanderhill, and R.B. Wattson: Proc. SPIE 143,114 (1978)

19.6 R.E. Griffiths, G. Polluci, A. Mak, S.S. Murray, D.A. Schwartz, and M.V. Zombeck: Proc. SPIE 244, 57 (1980)

19.7 R. Melen and D. Buss: Charge-Coupled Devices: Technology and Applications, IEEE Press (1977) 
19.8 J. Janesick, J. Hynecek, and M.M. Blouke: Proc. SPIE 290, 165 (1981)

19.9 R.A. Stern, K. Liewer, and J.R. Janesick: Rev. Sci. Instrum. 54, 198

19.10

W. Bradley: Proc R.E. Griffiths, S.S. Murray, M.V. Zombeck,

and

19.11 S. Marcus, R. Neison, and R. Lynds: Proc. SPIE 172, 207 (1979) 
4.12 The Design and Evaluation of Grazing Incidence Relay Optics

J.M. Davis, R.C. Chase, J.K. Silk, and A.S. Krieger

American Science and Engineering, Inc.

Cambridge, Massachusetts 02139

WGIFA. FAGE IS

DF POOR QUALITY 
